\title{
SC|A?
}

\section{Estratégias de uma pesquisa sobre culturas indígenas: $O$ uso do Whatsapp como recurso para produção de dados}

Nilva Heimbach ${ }^{1}$

\section{Resumo}

O artigo propõe argumentar sobre o uso do recurso WhatsApp como ferramenta possível para pesquisa em educação. Apresenta os passos percorridos em uma investigação sobre culturas indígenas na Disciplina de Arte, obrigatória em decorrência da Lei 11.645/2008. Constata-se que o recurso é apropriado para produção de dados, por oportunizara comunicação sincrônica ou assincrônica, favorecendo o diálogo com os entrevistados em tempos e espaços diferenciados, proporcionando que pesquisador e sujeitos da pesquisa estejam em campo em momentos distintos. O recurso tecnológico possibilitou discutir as percepções dos professores de Arte, suas propostas e as dificuldades encontradas com o tema culturas indígenas.

\section{Palavras-chave}

Pesquisa. Culturas indígenas. WhatsApp. Professores de Arte.

Recebido em: $14 / 04 / 2020$

Aprovado em: 25/06/2020

\footnotetext{
${ }^{1}$ Possui graduação em Educação Artística pela Universidade Federal de Mato Grosso do Sul (1989), graduação em Segunda Licenciatura em Pedagogia pela Faculdade Educacional da Lapa (2018), mestrado em Educação pela Universidade Católica Dom Bosco (2008) e doutorado em Educação pela Universidade Católica Dom Bosco (2019). Atualmente é professor convocado da Universidade Estadual de Mato Grosso do Sul e professor da Prefeitura Municipal de Campo Grande. Tem experiência na área de Artes, com ênfase em Educação Artística, atuando principalmente nos seguintes temas: cultura indígena, artesanato regional, ensino de arte, apreciação estética, exposição e interculturalidade, currículo, colonialidade.

Email:nilvahe@hotmail.com
} 


\section{Strategies of a research on indigenous cultures: The use of Whatsapp as a resource for data production}

\section{Abstract}

The article proposes to argue about the use of WhatsApp as a possible tool for research in education. It presents the steps taken in an investigation of indigenous cultures in the Discipline of Art, mandatory due to Law 11.645/2008. It is noted that the resource is appropriate for data production, as it allows for synchronous or asynchronous communication, favoring the dialogue with the interviewees at different times and spaces, allowing the researcher and research subjects to be in the field at different times. The technological resource made it possible to discuss the perceptions of Art teachers, their proposals and the difficulties encountered with the theme of indigenous cultures.

\section{Keywords}

Research. Indigenous cultures. Whatsapp. Artteachers. 


\section{Introdução}

Em 2019, ao escrever a tese ${ }^{2}$ que discutia as culturas indígenas e o ensino de arte, busquei refletir sobre as propostas e percepções dos professores de Arte. Esse tema já vinha sendo estudado desde longa data. Recordo que, na observação das práticas escolares, indagava: Como professores não indígenas apresentam em suas práticas pedagógicas as manifestações culturais indígenas? Essa indagação me impulsionou a refletir sobre as propostas e percepções de professores de Arte com a cultura indígena.

Na pesquisa, parti da complexidade que o trabalho com a cultura proporciona e que, no entanto, é tradutória, pois, como afirma Bhabha (2005, p. 314), "a tradução cultural dessacraliza as pressuposições transparentes da supremacia cultural e, nesse próprio ato, exige uma especificidade contextual, uma diferenciação histórica no interior das posições minoritárias”. Portanto, envolve processos de negociação, em ir além do encontro, recolocando lugares híbridos, articulando elementos antagônicos e contraditórios, retraçando as fronteiras.

Nesse sentido, "ir além", "cruzar fronteiras", de acordo com Silva (2005, p. 88), significa não respeitar os sinais que demarcam 'artificialmente' - os limites entre os territórios das diferentes identidades. Circunstâncias que submergem nas práticas escolares. Em sintonia com Bhabha (2005), com o entendimento de que se vive na "fronteira do presente", no momento de trânsito, o que sugere movimento, deslizamento, o ser permanece em contínuo processo de articulação com diferenças culturais, as quais já não são mais fixas. Fronteiras que sugerem possibilidades de "ir além", "viver além", deslocamentos em um mundo desigual e assimétrico. Há perigos na fixidez e no fetichismo de identidades e há deslocamentos que causam estranhamento perturba as relações estabelecidas, promovendo a negociação das diferenças.

Assim, lancei-me na pesquisa com o objetivo de discutir as relações estabelecidas em propostas curriculares entre o ensino de Arte e a diferença. $\mathrm{O}$

2 CULTURAS INDÍGENAS, ENSINO DE ARTE E A LEI 11.645/2008: possibilidades interculturais? UCDB/2019. 
campo empírico da pesquisa dialogou com as experiências e percepções de professores de Arte da rede púbica (municipal) que atuam na Educação Básica e com propostas que envolvem as culturas indígenas, tendo em vista o cumprimento da Lei 11.645/2008.

Comsubsídios em Candau (2008), refleti sobre a escola como "um espaço de cruzamento de culturas, fluido e complexo, atravessado por tensões e conflitos" (CANDAU, 2008, p. 15) para aprofundar na compreensão das relações estabelecidas no ensino e Arte que rompe com o caráter padronizador, homogeneizador e monocultural imposto na educação. Logo, as relações entre arte, escola e povos indígenas, propiciam reflexões sobre a escola como um espaço de encontro entre culturas, no caráter do respeito à diferença. $\mathrm{O}$ ensino de Arte, com propostas visando uma educação intercultural, pode contribuir como canal de negociação, abrir diálogo com a diferença, de modo específico, entre povos indígenas e não indígenas.

Nas perspectivas descritas, para discutir as culturas indígenas em propostas concebidas no ensino de Arte, uma das ferramentas utilizadas foi o WhatsApp. Assim, descrevo, neste artigo, os caminhos investigativos desenvolvidos na pesquisa sobre a relação entre o ensino de Arte e as culturas indígenas e a relevância do WhatsApp³ para a produção de dados.

\section{Culturas indígenas, Arte e tecnologia: caminhos possíveis para a pesquisa}

Na linguagem da arte, há criação, construção, invenção. O ser humano, por meio dela, forma, transforma a matéria oferecida pelo mundo da natureza e da cultura em algo significativo. Atribui significados a sons gestos, cores, com uma intenção, num exercício que mais parece um jogo de armar, um quebracabeça no qual se busca a forma justa. Vários caminhos são percorridos, várias soluções são experimentadas, num processo de ir e vir, um fazer/construir lúdico/estético que, embora comparada a um jogo, tem a diferença de que este jogo e suas regras são inventados enquanto se joga e por quem joga (MARTINS, PICOSQUE e GUERRA, 2010, p. 47/8).

3Aplicativo de mensagens instantâneas para uso em celulares, criado em 2009. Em 2015, passa a ser usado em computadores. 
Como a arte que cria e (re)cria, inventa, atribui significados, percorre caminhos lúdicos e estéticos, sociais e políticos, busquei indicações, caminhos para a pesquisa. Procurei alinhar as intenções de pesquisa com o recurso do aplicativo do WhatsApp, com a finalidade de investigar relatos de experiências sobre a cultura indígena. $\mathrm{O}$ recurso muitas vezes entendido como algo voltado para o lazer, para comunicados informais, com ele, busquei construir dados.

Participando de grupos de professores de Arte que compartilham suas experiências em diferentes recursos tecnológicos, percebi a potencialidade do contato na realidade virtual como canal de acesso a entrevistas/bate papos. Trago, portanto, reflexões indicando o potencial do uso do recurso WhatsApp para o desenvolvimento da pesquisa e a identificação de um grupo de professores de Arte para investigar o campo empírico.

Voltei, na pesquisa, meu olhar para Campo Grande, município com grande densidade demográfica com uma população estimada de 885.711 habitantes ${ }^{4}$,e que possui um crescente número de autodeclarados indígenas em contexto urbano. Nos dados do IBGE (2010), constava que a cidade possuía 5.898 habitantes declarados indígenas, dispersos em diversas localidades da cidade. No período da pesquisa, constava com aproximadamente 100 escolas municipais, muitas, atendendo alunado de diferentes etnias5. Constava também, de professores com lotações em diferentes escolas, levando o profissional a percorrer grandes deslocamentos. Compreendo, como Santos (2009), que "as características da população-alvo determinam a escolha das técnicas mais adequadas a cada situação" (2009, p.140). Considerando a realidade dos professores de Arte de Campo Grande/MS, a estratégia tecnológica pode ser eficaz para ir ao encontro de profissionais que desenvolvam propostas com a cultura indígena sem que interferisse na rotina deles. E, assim, elegi o WhatsApp como possibilidade de comunicação com os sujeitos da pesquisa.

4 Dados atualizados em: https://cidades.ibge.gov.br/brasil/ms/campogrande/panorama. Acesso em: jan. 2019.

5Campo Grande possui quatro aldeias urbanas e cinco bairros com grande concentração de indígenas que reivindicam a criação de aldeia urbana. Esses bairros são denominados pelos indígenas e reconhecidos pelo Conselho Municipal dos Direitos e Defesa dos Povos Indígenas de Campo Grande/MS como acampamentos indígenas. 
$\mathrm{Na}$ contemporaneidade, o contato virtual e o uso da tecnologia da informatização faz parte do uso diário. De modo particular, o uso do celular se tornou parte do cotidiano como ferramenta de comunicação e de registros, tanto escritos, de sons, de imagens fixas quanto de movimento. Uma multiplataforma que, de certo modo, aproxima as pessoas e as vivências, pois está ao alcance de muitos e facilita a transmissão de dados. Portanto, mídias que "têm propiciado maior qualidade e precisão metodológica às pesquisas científicas" (SANTOS, 2009, p.135). Percebendo os procedimentos como Santos (2009),

Partindo do delineamento da pesquisa, que envolve a elaboração de um plano geral de coleta e operacionalização dos dados, selecionam-se os métodos e técnicas a serem utilizados e as formas de aplicação. A estratégia multimetodológica possibilita integrar a técnica de observação com a de entrevista e questionário, entre outras. A sobreposição de procedimentos permite analisar a problemática e estudo a partir de diversos ângulos e dimensões (TANIA SANTOS, 2009, p.149).

Estabelecida a estratégia de contato, grupo de WhatsApp, organizei a sondagem e experimentação do instrumento, no sentido de verificar as possibilidades, limitações e implicações para a pesquisa.Para as entrevistas/bate papos on-line, elegi um grupo de WhatsApp do qual faço parte, identificado como Grupos Professores de Arte de Campo Grande ${ }^{6}$.

Pautei-me na escolha do grupo, adotando alguns critérios: os componentes trocam experiências; acesso aos participantes em diversos locais e horários variados;possibilidade de conversas descontraídas, sem rigidez formal. Critérios que desejavam mapear experiências de produções sobre a temática indígena por professores de Arte em diferentes realidades.

Concebo, como Félix (2014),que as redes sociais virtuais como possíveis instrumentos "úteis para a produção de material empírico de pesquisa [...]" (FÉLIX, 2014, p. 135). Ela ressalta que a comunicação on-line pode ser organizada de forma síncrona (em tempo real) ou assíncrona (não sendo imprescindível a conexão em tempo real), uma vez que, na internet, no uso de

${ }^{6} \mathrm{O}$ grupo foi criado em outubro de 2014 por uma professora de Arte com o propósito de compartilhar experiências. O critério de escolha do grupo: a) o grupo já possui o hábito de trocarinformações; b) os componentes são professores de Arte; c) os profissionais trabalham em diferentes escolas públicas de Campo Grande. 
redes sociais, as fronteiras de tempo e espaço se combinam num mundo on-line e off-line. Além disso, ela destaca que,no uso da internet,"estar perto7 e estar longe podem ter significados similares e diferentes, dependendo da situação e, às vezes, de um clique no mouse e,ainda, as expressões da realidade virtual e presencial,que podem ter múltiplos significados. Com as vantagens, desafios e limitações dos procedimentos, indica a autora que "o material empírico é produzido por escrito, excluindo a necessidade de transcrição das entrevistas; permite acessar participantes de diferentes lugares" (FÉLIX, 2014, p. 138), oportunizando a ubiquidade. No entanto, como limite, apenas os participantes do grupo, pessoas com acesso à internet podem participar.

Com a determinação do uso da estratégia de produção de dados,discuti, a partir dos Estudos Culturais, da Modernidade/Colonialidade, relações epistêmicas, políticas, sociais e pedagógicas entre eles. Tratei de relações de poder, respeito ao outro e como são traduzidas nas práticas pedagógicas no ensino de Arte. Referências "que nos ajudam pensar de modo diferente do que já foi pensado o nosso objeto, que nos possibilitam usar o "e" da ligação, da soma e da multiplicidade" (PARAISO, 2014, p.38).

Por conseguinte, no diálogo com a perspectiva descrita, relacionando o ensino de Arte e a Lei 11645/2008, procurei cunhar que as propostas desenvolvidas no ensino de Arte têm possibilidades de proporcionar debates sobre e com as manifestações culturais indígenas em diálogo com a interculturalidade, sem apontar para uma cultura mais valorada e sim para diferenças integrantes das diversas identidades. Entendo, pois, que a interculturalidade aproxima os diversos sujeitos que transitam no ambiente escolar, sem a busca da homogeneização.

\section{Fase exploratória: o instrumento de pesquisa}

Em novembro de 2017, iniciei a relação investigativa com o grupo ${ }^{8}$ com a finalidade de testar o instrumento de pesquisa. Estratégia para verificar a

7 Grifos da autora.

${ }^{8} \mathrm{Na}$ fase exploratória, constava com 26 componentes, incluindo a pesquisadora. 
aceitação dos participantes e a viabilidade do caminho investigativo. Expus os objetivos da pesquisa, convidando-os para narrarem suas propostase percepções, com e sobre as culturas indígenas. Compartilhei o convite e aguardei 48 horas.

Dos professores participantes, 10 (dez) declararam disponibilidade em colaborar com a pesquisa. Com estes, fiz o contato individual, passei a comunicação para o aplicativo WhatsApp privado e não mais lançando as questões para todos os participantes do grupo. No decorrer dos contatos privados, foi possível perceber que,dos 10 (dez) professores colaboradores iniciais: - um não estava trabalhando em sala de aula com a Disciplina Arte; dois não desenvolveram o tema;- um, pouco abordou o tema e não tem registros;- um afirma que trabalhou com o tema no Ensino Médio da rede Estadual (Projeto MS 40 anos) e na rede privada no $3^{\circ}$ ano da Fundamental I, seguindo a Apostila adotada na escola, porém, estava sem fotografias no celular, mas ofereceu uma data futura para disponibilizar os registros;- e uma afirmou que trabalhou com o $5^{\circ}$ ano da rede municipal, mas sem registros fotográficos.Portanto, esses profissionais (6 professores), por não atenderem aos objetivos investigativos propostos, não participaram na continuidade da etapa de sondagem da pesquisa, ou porque não desenvolveram propostas ou porque não tinham registros sobre o desenvolvimento de suas experiências.

Dando continuidade ao processo investigativo, elegi 4 sujeitos com suas propostas para o teste do recurso WhatsApp: a) os que desenvolveram propostas com a cultura indígena; b) os que enviaram registros fotográficos sobre o tema em questão dentro do prazo estabelecido; c) profissionais que não são indígenas; d) trabalham em escolas públicas.

Com os resultados obtidos, optei por:

a. continuar a pesquisa com o uso do WhatsApp: O aplicativo WhatsApp, demonstrou ser canal de comunicação eficiente, viável para a pesquisa com professores de Arte;

b. permanecer com os professores de Arte do Grupo Professores de Artes CG. Os professores que trabalham com a temática indígena; 
c. Ampliar o tempo para o contato com os profissionais que trabalham com a cultura indígena;

d. Conversar com professores que relatam a experiência com a temática, porém não têm registro com imagens.

Compreendi, a partir dos estudos de Félix (2014), que é possível "estar em campo"; que, estar on-line e off-line leva a processos interligados, a comunicação é realizada sem a necessidade do tempo real, e, por isso, "pensar que ir a campo é um termo que não dá conta de suas dimensões. Assim, parece que estar em campo é o mais apropriado" (FÉLIX, 2014, p. 139), pois, em qualquer momento, as comunicações podem ser estabelecidas. Portanto, o momento de exploração termina, mas o instrumento WhatsApp se tornou companheiro da pesquisa.

Entrevistados 2018: profissionais colaboradores da pesquisa

Ler uma imagem é saboreá-la em seus diversos significados, criando distintas interpretações (PIlLAR, 1999, p.17).

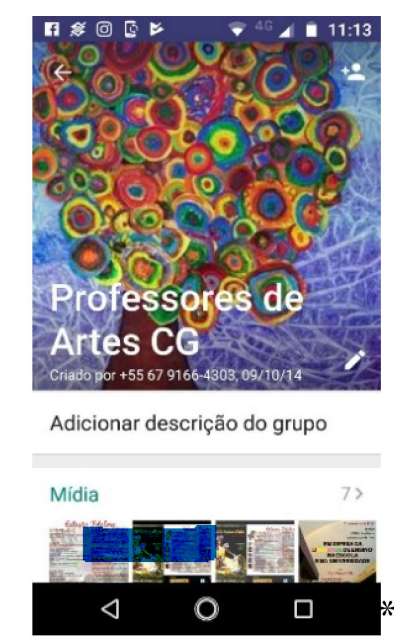

Figura 1. Grupo WhatsApp Professores de Artes CG Fonte: Acervo Pessoal (2018)

Um grupo de WhatsApp, uma imagem. Um grupo de professores de Artes que se inspira na obra de Kandinsky para layout de sua identificação. Na imagem (Fig.1), uma árvore abstrata, composta de círculos coloridos de diferentes tamanhos e cores. O fundo de azul intenso com hachuras brancas, sugerindo volume e movimento. 
O tronco/arte sustenta os círculos professores, e cada qual carrega sua identidade de cores que se movimentam no mundo de possibilidades... Possibilidades para o ensino da Arte. Possibilidades que podem ser expressas nas propostas e intervenções que professores e professoras de Arte realizam, com suas diferenças e semelhanças, buscando refletir sobre algumas possibilidades de realizações de propostas sobre e com as culturas indígenas, em escolas não indígenas e por professores não indígenas.

De tal modo, em maio/2018, já ciente de que a via de comunicação com o WhatsApp é possível, retomo o contato com o Grupo de Professores de Artes CG para uma nova seleção de sujeitos, quando procuro não apenas ir a campo, mas estar em campo (FÉLIX, 2014), onde o material empírico é produzido em diferentes lugares e a comunicação é estabelecida de forma sincrônica ou assincrônica possibilitando aos sujeitos da pesquisa participarem em horários que lhes forem convenientes. Feita a escolha do campo empírico, busquei estabelecer critérios $^{9}$ de seleção dos sujeitos da pesquisa.

Lancei o convite de participação e, conforme estabeleciam o contato, passei a conversar no número de celular privado. Foi usado, pois, como recurso e canal de comunicação, o WhatsApp, multiplataforma, tanto com texto escrito, quanto com mensagem de voz, transcrito com o recurso do Transcriber, e-mail, fotografias e vídeos.

Apontando para os objetivos da pesquisa, novamente, difundi o convite no grupo, apresentando-me, informando o foco dos estudos e a linha de pesquisa:

Caros colegas, bom dia!

Meu nome é XXX, sou professora do IESF/UEMS e efetiva da prefeitura municipal, cursando o doutorado no PPGE - UCDB, na linha de pesquisa Diversidade Cultural e Educação Indígena e participo do GPEIN (Grupo de Pesquisa Educação e Interculturalidade). Pesquiso a relação entre o ensino de arte e a cultura indígena no espaço escolar.

9Para atender aos objetivos da pesquisa, entrevistar Professores de Arte - com experiência na Educação Básica, com relatos de experiências de propostas relativas à cultura indígena - que aceitassem dialogar sobre suas experiências desenvolvidas nos espaços escolares públicos e que não se declarassem indígenas. 
No ano passado, enviei convite solicitando a colaboração para minha pesquisa de doutorado, vários professores me ajudaram. Agora estou em outra etapa da pesquisa e preciso novamente da colaboração dos colegas, que relatem suas experiências. Como resultado, espero contribuir com o fortalecimento de nossa categoria e com a divulgação de nossas propostas pedagógicas. Quem pode colaborar? Em caso afirmativo, me dê um sinal que entrarei em contato. Em tempo: meu foco é apenas este grupo. Obrigada pela colaboração! (21-05-18).

Nessa nova etapa, dos 25 (vinte e cinco), apenas 6 (seis) professores não responderam ao comunicado inicial; totalizando 19 professores que responderam ao contato inicial. No entanto, 3 (três) justificaram o motivo de não participar e 1 (um) solicitou que enviasse por e-mail, porém não deu continuidade na comunicação. Reduzindo os participantes a 15 professores colaboradores. Dos três que justificaram: um professor está trabalhando com cerâmicas com adultos e não trabalha a temática indígena; o segundo, fazendo artesanatos e não está atuando em sala de aula e o terceiro, atualmente, está realizando uma pós (especialização), com pouco tempo para outras atividades, este último, colaborou na etapa passada. Portanto,

- Lancei o convite para os demais 25 componentes;

- Responderam: 19 componentes;

- Não aderiram: 4 componentes

- Aderiram: 15 componentes.

Em Junho/2018, passei a me dedicar aos 15 (quinze) professores do grupo que relataram suas experiências e percepções. No decorrer das semanas, várias conversas surgiram. Alguns estavam desejosos para relatar outras experiências formidáveis, referentes a outros conteúdos, mas que não eram a proposta deste estudo. Observei que alguns (nove professores) não se sentiam confortáveis em relatar pormenores de seus trabalhos referentes às culturas indígenas. Não insisti. Agradeci a participação, porém optei por aqueles 6 (seis) professores que demonstraram interesse de compartilhar e divulgar as suas propostas sobre o tema.

Dos 4 (quatro) que participaram da testagem do instrumento no ano anterior (bate-papo via WhatsApp), dois permaneceram na seleção atual.Selecionei professores colaboradores que estivessem desejosos de relatar suas 
experiências. Concordando com Fanon (2008, p.33), quando ele afirma que "falar é existir absolutamente para o outro" (FANON, 2008, p. 33), falar das experiências pedagógicas para uma pesquisa é levá-la para outros locais, existir em outros locais.

Portanto, para esta etapa da pesquisa, contei comexperiências de 6 (seis) professores de Arte, com trajetórias e propostas distintas, os quais não foram identificados por seus nomes e sim por círculos cromáticos, com suas cores de preferência. Círculos que estão no layout de identificação do grupo, círculos que inspiram movimento, continuidade, modificação. Círculos coloridos que movimentam e que o olhar do outro nem sempre consegue captar todas as cores neles contidas. Em sintonia com Gauthier (2012, p.65), entendo que "a vida muda sempre, e o que estava estabilizado numa época, hoje já é diferente, obedecendo a outras leis”. Assim, são os círculos cromáticos, assim são as propostas desenvolvidas com sujeitos híbridos.

Após a triagem dos sujeitos da pesquisa, permaneci com bate-papos via WhatsApp. Recebi relatos diversos, com e sem fotografias. Os retornos eram realizados nos bate-papos do WhatsApp, com textos escritos, emojis e áudios; em alguns momentos, a comunicação estabelecida foi via e-mail. Os questionamentos foram divididos em quatro momentos, cada qual agregando informações para discussão dos objetivos do estudo. Ressalto que esses questionamentos não eram exatamente iguais, pois dependiam do desenvolvimento das conversas, os quais não eram respondidos instantaneamente. Assim, os diálogos seguiam caminhos inesperados, uma vez que:

Fazer pesquisa em Educação é "encontrar o 'outro" na dinâmica dessas 'pontes'. É, sabendo que a linguagem é incompletude, investir em uma escuta atenta das narrativas das experiências dos nossos interlocutores e interlocutores, posicionando-nos dialogicamente nessa relação (PASSOS, 2014, p.228).

O primeiro momento oportunizou a seleção dos sujeitos, os entrevistados colaboradores, conforme já assinalado anteriormente, e indicou como as representações das culturas indígenas são apresentadas e traduzidas. Foi a 
etapa mais descontraída e calorosa, e o número de participantes foi bem maior do que nas etapas anteriores.

No segundo momento, as conversas incidiram nos diversos perfis dos colaboradores, principalmente dos que sentiam o desejo de discorrer sobre as suas propostas em relação à cultura indígena. Conversamos com a pretensão de oferecer alguns traços e mapear o perfil do professor de Arte da rede pública, que, no momento da pesquisa, estava disposto a dialogar sobre o tema. Discorremos e sinalizamos alguns dos aspectos da identidade do profissional colaborador.

No terceiro momento, as conversas foram referentes ao entendimento de como as etnias/culturas/saberes indígenas e as suas manifestações são apresentadas por tais profissionais. Etapa que atribui como a arte e a cultura indígena são discutidas no espaço escolar, sinalizando marcadores para perceber se as relações estabelecidas oferecem indicativos de propostas interculturais. No caso de dúvidas, inseri um quarto momento, buscando entender as propostas realizadas pelos professores.Portanto, as conversas com os professores colaboradores "círculos cromáticos" procuravam indicar marcadores de interesse pelo tema culturas indígenas.

Desse modo, para cada professor colaborador participante, integrante da árvore/símbolo/círculo, foi solicitado que identificasse as cores de suas preferências, para identificá-lo como círculo da árvore/símbolo, demonstrando a policromia de sua identidade múltipla, híbrida. Assim, como afirmado, para preservar a identidade dos entrevistados, os profissionais colaboradores foram descritos com nomes fictícios, como círculos coloridos. Na construção dos diversos perfis, os profissionais colaboradores, círculos coloridos, escolheram seus codinomes, com suas cores preferidas. Ei-los: Laranja-turquesa-roxo; Vermelho-Vermelho-Azul; Vermelho-Azul-Verde-Vermelho; Vermelho-AzulVermelho; Turquesa-Vermelho-Azul e Vermelho-Preto.

Cada proposta foi expressa de uma maneira, segundo o perfil de cada profissional. Alguns com riqueza de detalhes,com fotografias, outros de forma 
sucinta, quase sem registro. No entanto, todos discorriam com empolgação, comunicavam suas práticas como algo especial na realização do seu exercício profissional.

Nas conversas, notei tensionamentos entre os desejos expressos pelos professores e as realidades existentes. Negociações permanentes.Ressalto que o aspecto interpretativo para a produção dos dados é um momento delicado e respeitoso com os colaboradores.E aqui, empresto o termo "confeto" de Gauthier (2012) para interpretar as experiências propostas, com misturas de conceitos e afetos. Entendo como Kincheloe (2007, p.44) que "todos estão destinados a ser intérpretes que analisam o cosmo de dentro de suas fronteiras e com os seus tapa-olhos. Para pesquisar, temos que interpretar; na verdade, para viver, temos que interpretar". Assim, na pesquisa, discorri sobre minhas interpretações relacionadas a propostas de professores de Arte com a cultura indígena.

Nos depoimentos, encontrei algumas fronteiras deslizantes. As culturas indígenas podem ser trabalhadas no decorrer do ano letivo, mas predomina em datas especiais. Pode ocorrer em todos os anos escolares, mas predomina nos anos iniciais, $4^{\circ}$ e $5^{\circ}$ anos. Oferece a sensação de silenciamento nos anos finais, uma vez que os conteúdos estão relacionados à História da Arte Ocidental, indicando a imposição de um currículo colonizador, com saberes eurocentrados.

\section{Considerações Finais}

Conforme anunciado, para o desenvolvimento da pesquisa questionei como as culturas indígenas são traduzidas nos espaços escolares por professores não indígenas, uma vez que compreender a tradução oportuniza negociações com a diferença e vivências interculturais. Busquei, assim, discutir as relações entre o ensino de arte, a diferença, saberes indígenas; debatendo sobre as práticas escolares e a Lei 11.645/2008. Delineei possibilidades de caminhos investigativos para discutir as culturas indígenas no ensino de arte. Como estratégia metodológica, descrevi a sondagem do aplicativo WhatsApp, e, para a 
travessia da pesquisa, assegurei-mede que o recurso é viável para compartilhar experiências com e sobre as culturas indígenas.

Nas narrações dos profissionais, procurei analisar o ensino de arte como espaço de negociações e tensões, observando, assim, as relações estabelecidas, a procura pela interculturalidade, os silenciamentos e os processos que se alinham com o pensamento colonial.

Os resultados sugerem a preocupação com a diferença, desejos de propostas com possibilidades interculturais. Porém, os professores colaboradores, aqui chamados de "círculos cromáticos, relataram dificuldades por falta de acesso a informações pertinentes aos saberes indígenas". Há carência de capacitações/formações referentes à Lei 11.645/2008, e encontraram barreiras com o currículo eurocentrado/colonizado; escasseiam materiais de apoio para a realização de propostas artísticas. Além disso, o tema se encontra, na maioria das vezes, datado em 19 de abril, no Dia do Índio.

Apesar dessas dificuldades, os professores de Arte manifestaram empenhos em ir além do aspecto estético das culturas indígenas. Preocupam comas identidades, as realidades sociais e as relações de poder existentes, o que me fez perceber que o ensino de Arte, sobretudo, na Educação Básica e com propostas visando uma educação intercultural, pode contribuir como canal de negociação, abrir diálogo com a diferença.

\section{Referências}

BHABHA, Homi. O local da cultura. Belo Horizonte: Editora da UFMG, 2005.

BRASIL. Lei ${ }^{\circ} 11.645$, de 10.03.08. Altera a Lei no 9.394, de 20 de dezembro de 1996, modificada pela Lei no 10.639, de 9 de janeiro de 2003, que estabelece as diretrizes e bases da educação nacional, para incluir no currículo oficial da rede de ensino a obrigatoriedade da temática "História e Cultura Afro-Brasileira e Indígena”. Brasília. 2008.

CANDAU, V. M. F. Multiculturalismo e educação: desafios para a prática pedagógica. In: MOREIRA, Antônio Flávio. CANDAU, Vera Maria (Orgs.). Multiculturalismo: diferenças culturais e práticas pedagógicas. 2. ed. Petrópolis, RJ: Vozes, 2008, p. 13-37. 
FANON, Frantz. Pele negra. Máscaras brancas. Rio de Janeiro: Editora Fator, 2015.

FÉLIX, Jeane. Entrevistas on-line ou algumas pistas de como utilizar batepapos virtuais em pesquisas na educação e na saúde. In: MEYER, Dagmar Estermann; PARAÍSO, Marlucy Alves (Orgs.). Metodologias de pesquisas póscríticas em Educação. Belo Horizonte: Mazza Edições, 2014, p.235-154.

GAUTHIER, Jacques. Carta aos caçadores de saberes populares. Conversação com Elisa Pereira Gonçalves. In: COSTA, Marisa Vorraber (Org.).Educação popular hoje. São Paulo: Edições Loyola, 1998, p.181-212.

GAUTHIER, Jacques. $O$ oco do vento: metodologias da pesquisa sociopoética e estudos transculturais. Curitiba: CRU, 2012.

HEIMBACH, Nilva. Culturas indígenas, ensino de Arte e a Lei 11.645/20o8: possibilidades interculturais? Tese (Doutorado em Educação). Universidade Católica Dom Bosco, Campo Grande, 213 p. 2019.

IBGE. Os indígenas no Censo Demográfico 2010. Brasília, 2010. Disponível em: http://www.ibge.gov.br/indigenas/indigena_cens02010.pdf. Acesso em:jan. 2019.

KINCLHELOE, J. L.; O poder da bricolagem: ampliando os métodos de pesquisa. In: KINCLHELOE, J. L.; KATHLEEN, S. B; Pesquisa em Educação: Conceituando a bricolagem. São Paulo: ARTMED. 2007, p.15-37.

MARTINS, Mirian Celeste; PICOSQUE, F. D.Giza; GUERRA, M.T.T.Teoria $e$ prática do ensino de Arte: a língua do mundo. São Paulo: FTD, 2010.

PARAÍSO, Marlucy. Metodologias de pesquisas pós-críticas em Educação e currículo: trajetórias, pressupostos, procedimento e estratégias analíticas. In: MEYER, Dagmar Estermann. e PARAÍSO, Marlucy Alves (Orgs.). Metodologias de pesquisas pós-críticas em Educação. Belo Horizonte: Mazza Edições, 2014, p.25-47.

PASSOS, M. C. P. Encontros cotidianos e a pesquisa em Educação: relações raciais, experiência dialógica e processos de identificação. Educar em Revista, Curitiba, Brasil, n. 51, p. 227-242, jan./mar. 2014.

RICHTER, Ivone Mendes. Interculturalidade e estética do cotidiano no ensino das artes visuais. Campinas: Mercado de Letras, 2003.

SANTOS, Tania S. Do artesanato intelectual ao contexto virtual: ferramentas metodológicas para pesquisa social. Sociologias. Porto Alegre, ano 11, n. 21, jan./jun., p.120-156, 2009.

WALSH, Catherine. Interculturalidade Crítica e Pedagogia Decolonial: in-surgir, re-existir e re-viver. In: CANDAU, Vera Maria (Org.). Educação Intercultural na América Latina: entre concepções, tensões e propostas. RJ: Ed.7 Letras, 2009, p.12-41. 